\title{
Influence of some insecticide sequences on the injurious insect- pests of cotton plants
}

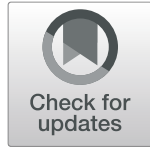

Seham M. Ismail

\begin{abstract}
Background: Cotton growers in Egypt have experienced severe economic loss from cotton bollworms, especially the pink bollworm, Pectinophora gossypiella (Saund.), and spiny bollworm, Earias insulana (Boisd.), so a field experiment was carried out to determine efficiency of eight insecticides in their seven sequence programs to evaluate the best solution for cotton bollworms problems, during two successive seasons.

Results: The highest reduction percentages are effective sequences 7 and 5 (gave about 90\% infestation reduction) were started and mediated with new insecticides, and when started and mediated with spinosad, or with cypermethrin, sequences 2 and 3 (gave about 86\%) respectively, also, the repetition of the same insecticide twice in a regimen might increase, might decrease, or has no effect on the insecticide efficiency against cotton bollworms. The tested sequence programs showed high protection for green bolls with protection percentages ranged from 66.1 and $67.2 \%$ (sequence 1) to 92.6 and $94.1 \%$ (sequence 7) during two seasons respectively. On the other hand, the superiority in yield loss reduction can arrange in the following descending order: sequences $7,5,2,3,6,4$, and 1 respectively.

Conclusions: From this study, it can be concluded that the importance of sequence insecticide programs included new groups of insecticides to directing tactics to fight against resistance development and used this sequence programs to restore susceptibility for conventional insecticides. This study devoted to obtaining the optimum regimen of insecticides that give low reduction of cotton bollworms' infestation and high cotton yield.
\end{abstract}

Keywords: Cotton bollworms, Pectinophora gossypiella, Earias insulana, Pesticide sequence programs

\section{Background}

Cotton growers in Egypt have experienced severe economic loss from cotton bollworms, especially the pink bollworm, Pectinophora gossypiella (Saund.), and spiny bollworm, Earias insulana (Boisd.), which spend most of their life cycles inside the bolls and are considered to be a great menace because they can destroy the plants in a few days. Bollworms mainly feed on fruiting parts of cotton, resulting in considerable losses both in quality and quantity and $20-60 \%$ damage to and a decrease in the market value of fiber. The larvae of E. insulana attack soft and growing tissues, especially terminal bud of the main stem, flower buds, and bolls, which ultimately shed. Therefore, plant protection has now become a necessity of the most important components of a profitable farming system

\section{Correspondence: Dr.Sehammansour1@gmail.com}

Central Agricultural Pesticides Laboratory, Agricultural Research Center, Giza, Egypt to overcome the losses, keep the level of the infestation under the economic threshold, and to obtain the good yield (Amin and Gergis 2006). Control of these pests have depended exclusively on conventional insecticides which have an important role in management programs to control cotton bollworms; as a result, occurred high level of resistance to these insecticides in cotton bollworms and other pests of cotton (Ramsubramanian 2004) have resulted in renewed interest in the farmers for using new group of insecticides available in the Egyptian markets (Gunning and Devonshire 2002). In order for the cotton bollworms control program to be successful, it has to rely on the use of insecticides belonging to different groups in certain sequences, application time, and spraying interval (Abd El-Mageed et al. 2007).

Newer chemistries of pesticides have raised the hopes for better management of destructive pest in many areas. Therefore, an attempt was made to study the evaluation
Springer Open

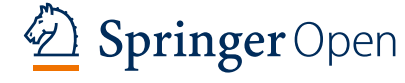

(c) The Author(s). 2019 Open Access This article is distributed under the terms of the Creative Commons Attribution 4.0 International License (http://creativecommons.org/licenses/by/4.0/), which permits unrestricted use, distribution, and reproduction in any medium, provided you give appropriate credit to the original author(s) and the source, provide a link to the Creative Commons license, and indicate if changes were made. 
of programs consisting of seven different insecticide sequences, including new groups of insecticides for the control of cotton bollworms (pink and spiny bollworms) in cotton crop, and the aim of our study was concentrated on obtaining the optimum regimen of insecticides which gives a reduction of the infestation with cotton bollworms, and an increase of the cotton yield.

\section{Materials and methods \\ Chemicals}

The evaluated insecticides, common names, and rates are introduced in Table 1.

\section{Field trials}

The experiments were conducted during the cotton growing seasons 2017 and 2018 in El-Beheira Governorate at Kafr El-Dawar district. The tested insecticides are listed in Table 1. The evaluated insecticides were used in seven sequences as follows: sequence no. (1) of profenofos, profenofos, cypermethrin, and cypermethrin; sequence no. (2) spinosad, profenofos, spinosad, and carbosulfan; sequence no. (3) of cypermethrin, profenofos, cypermethrin, and carbosulfan; sequence no. (4) carbosulfan, carbosulfan, cypermethrin, and cypermethrin; sequence no. (5) acetamiprid, cypermethrin, chlorfenapyr, and profenofos; sequence no. (6) of profenofos, spinosad, cypermethrin, and carbosulfan; and sequence no. (7) of cypermethrin, emmamectin benzoate, indoxacarb, and profenofos. The fields were cultivated with cotton variety "Giza 70"; the average percent infestation in all experimental field plots was monitored till that reached $7 \%$ at zero time of application. The experimental area was 2.5 feddans, and it was divided into plots of 2 Kirrats each, and the treatments were arranged in complete random plots with four replicates which were sprayed four times (every 2 weeks) using a ground motor sprayer at the rate of 400 liter per feddan.

Table 1 Recommended and used doses of the tested insecticides

\begin{tabular}{|c|c|c|}
\hline Pesticides & Group & $\begin{array}{l}\text { Rate of } \\
\text { application } \\
\text { (feddan) }\end{array}$ \\
\hline Acetamiprid 20\% SP (Acetamor $\left.{ }^{\circledast}\right)$ & Neonicotinoids & $100 \mathrm{~g}$ \\
\hline Chlorfenapyr 24\% EC (Pyricide ${ }^{\circledast}$ ) & Halogenated pyrroles & $300 \mathrm{~cm}^{3}$ \\
\hline Spinosad 24\% SC (Spintor ${ }^{\oplus}$ ) & Bio-insecticides & $50 \mathrm{~cm}^{3}$ \\
\hline $\begin{array}{l}\text { Emmamectin benzoate } \\
0.5 \% \text { EC }\left(\operatorname{Radic}^{\top}\right)\end{array}$ & Avermectains & $800 \mathrm{~cm}^{3}$ \\
\hline Indoxacarb 30\% WG (Abizo ${ }^{\oplus}$ ) & Oxadiazine & $125 \mathrm{~cm}^{3}$ \\
\hline Carbosulfan 24.7\% SC $\left(\right.$ Marshal $\left.^{\oplus}\right)$ & Carbamate & $600 \mathrm{~g}$ \\
\hline Cypermethrin 20\% EC (Cyparko $\left.{ }^{\oplus}\right)$ & Pyrethroids & $300 \mathrm{~cm}^{3}$ \\
\hline Profenofos 48\% EC (Dora $\left.{ }^{\oplus}\right)$ & O.P. & 1 liter \\
\hline
\end{tabular}

\section{Laboratory examination}

For each treatment, we took samples of 100 green bolls per treatment ( 25 bolls per each replicate) before spraying time and weekly after insecticide application. Percentage of infestation by pink bollworm, P. gossypiella, and that of spiny bollworm, E. insulana, was determined in the laboratory by dissection of bolls and checking the bolls externally and internally. The percent reduction of pink bollworm or spiny bollworm infestation was calculated according to Henderson and Tilton (1955).

\section{Estimation of cotton yield loss}

Randomly, one hundred cotton plants were chosen from each treatment to study the cotton yield loss caused by bollworms. The following method, according to Hassan (2007), was used to estimate the cotton yield losses. The complete opened bolls $(A)$, the $2 / 3$ opened bolls $(B)$, the $1 / 3$ opened bolls, the dry bolls $(D)$, and the unopened green bolls $(E)$ were counted; then, the expected open bolls $(F)$ were calculated as follows: $F=A+B+C+D+E$

The true opened bolls $(G)=A+(B \times 2 / 3)$

$$
+(C \times 1 / 3)
$$

The number of unopened bolls $(H)=F-G$

$$
\text { The } \begin{aligned}
\operatorname{loss} \%(I) & =(H / F) \times 100 \text { or } I \% \\
& =(1-G / F) \times 100
\end{aligned}
$$

\section{Statistical analysis}

All biological aspects were analyzed using one-way ANOVA by SPSS 13.0 (SPSS, 2004). Tukey's honestly significant difference (HSD) studentized range test was used to determine the probability level to compare the differences among some parameter means $(P<0.05)$.

\section{Results \\ Efficacy of tested sequences of insecticides against Pectinophora gossypiella during two seasons}

The elucidated data in Table 2 show the variation between the weekly calculated reduction percentages of the pink bollworm, $P$. gossypiella, during the application of seven suggested sequences of certain compounds. The percentages of infestation reduction of all tested compounds were more in the first week after treatment than that in the second week throughout the four sprays. In general, all the tested sequences resulted in an appreciable reduction in $P$. gossypiella infestation; as compared with control, the infestation reduction percentages were more than $72 \%$ during the two seasons. Regarding the second spray, the application of profenofos after cypermethrin caused 88.7 and $86.3 \%$, while after spinosad caused 90.5 and $89.7 \%$ reduction of infestation for both seasons 2017 and 2018, respectively. The statistical 
Table 2 Infestation reduction percentage of Pectinophora gossypiella during two seasons

\begin{tabular}{|c|c|c|c|c|c|c|c|c|c|c|c|c|c|}
\hline \multirow[t]{3}{*}{ Treatments } & \multicolumn{13}{|c|}{ Percentage of infestation reduction } \\
\hline & \multicolumn{2}{|c|}{ First spray } & \multicolumn{4}{|c|}{ Second spray } & \multicolumn{3}{|c|}{ Third spray } & \multicolumn{3}{|c|}{ Fourth spray } & \multirow{2}{*}{$\begin{array}{l}\text { General } \\
\text { mean }\end{array}$} \\
\hline & $\begin{array}{l}1^{\text {st }} \\
\text { week }\end{array}$ & $\begin{array}{l}2^{\text {nd }} \\
\text { week }\end{array}$ & Mean & $\begin{array}{l}1^{\text {st }} \\
\text { week }\end{array}$ & $\begin{array}{l}2^{\text {nd }} \\
\text { week }\end{array}$ & Mean & $\begin{array}{l}1^{\text {st }} \\
\text { week }\end{array}$ & $\begin{array}{l}2^{\text {nd }} \\
\text { week }\end{array}$ & Mean & $\begin{array}{l}1^{\text {st }} \\
\text { week }\end{array}$ & $\begin{array}{l}2^{\text {nd }} \\
\text { week }\end{array}$ & Mean & \\
\hline \multicolumn{14}{|l|}{ Season 2017} \\
\hline \multirow[t]{2}{*}{ Sequence 1} & \multicolumn{3}{|c|}{ Profenofos } & \multicolumn{3}{|c|}{ Profenofos } & \multicolumn{3}{|c|}{ Cypermethrin } & \multicolumn{3}{|c|}{ Cypermethrin } & \\
\hline & 78.3 & 75.0 & $76.7 \mathrm{~b}$ & 80.0 & 79.0 & $79.5 b$ & 72.4 & 70.9 & $71.7 \mathrm{~b}$ & 70.6 & 68.5 & $69.6 c$ & $74.4 b$ \\
\hline \multirow[t]{2}{*}{ Sequence 2} & \multicolumn{3}{|c|}{ Spinosad } & \multicolumn{3}{|c|}{ Profenofos } & \multicolumn{3}{|c|}{ Spinosad } & \multicolumn{3}{|c|}{ Carbosulfan } & \\
\hline & 90.4 & 85.0 & $87.7 a$ & 92.0 & 89.0 & $90.5 a$ & 82.9 & 80.0 & $81.5 a$ & 85.8 & 83.0 & $84.4 a$ & $86.0 a$ \\
\hline \multirow[t]{2}{*}{ Sequence 3} & \multicolumn{3}{|c|}{ Cypermethrin } & \multicolumn{3}{|c|}{ Profenofos } & \multicolumn{3}{|c|}{ Cypermethrin } & \multicolumn{3}{|c|}{ Carbosulfan } & \\
\hline & 84.0 & 81.0 & $82.5 a$ & 91.4 & 86.0 & $88.7 a$ & 83.6 & 80.2 & 81.9a & 82.6 & 78.0 & 80.3a & $83.4 a$ \\
\hline \multirow[t]{2}{*}{ Sequence 4} & \multicolumn{3}{|c|}{ Carbosulfan } & \multicolumn{3}{|c|}{ Carbosulfan } & Cyper & thrin & & Cyperr & thrin & & \\
\hline & 82.9 & 77.1 & $80.0 \mathrm{a}$ & 70.3 & 66.3 & $68.3 c$ & 77.4 & 71.0 & $74.2 b$ & 81.0 & 76.2 & $78.6 \mathrm{~b}$ & $75.3 b$ \\
\hline Sequence 5 & Acetan & & & Cyper & hrin & & Chlorf & apyr & & Profen & & & \\
\hline & 93.2 & 92.0 & $92.6 a$ & 82.6 & 80.4 & $81.5 a$ & 90.2 & 87.0 & $88.6 a$ & 93.3 & 89.0 & $91.2 \mathrm{a}$ & $88.5 a$ \\
\hline Sequence 6 & Profen & & & Spinos & & & Cyperr & thrin & & Carbos & & & \\
\hline & 88.1 & 84.4 & 86.3a & 84.3 & 82.6 & $83.5 a$ & 81.5 & 79.1 & $80.3 a$ & 76.7 & 75.7 & $76.2 b$ & $81.6 a$ \\
\hline Sequence 7 & Cypern & thrin & & Emma & ctin ber & & Indoxa & & & Profen & & & \\
\hline & 93.0 & 89.7 & $91.4 a$ & 96.4 & 95.0 & $95.7 a$ & 94.5 & 93.2 & 93.9a & 93.7 & 91.2 & $92.5 a$ & $93.4 a$ \\
\hline Season 2018 & & & & & & & & & & & & & \\
\hline Sequence 1 & Profen & & & Profen & & & Cyperr & thrin & & Cyperr & thrin & & \\
\hline & 81.8 & 79.2 & $80.5 a$ & 77.7 & 68.3 & $73.0 \mathrm{~b}$ & 71.5 & 68.5 & $70.0 \mathrm{~b}$ & 67.1 & 64.8 & $66.0 \mathrm{c}$ & $72.4 b$ \\
\hline Sequence 2 & Spinos & & & Profen & & & Spinos & & & Carbos & & & \\
\hline & 93.0 & 90.7 & 91.9a & 90.9 & 88.5 & $89.7 a$ & 81.6 & 79.0 & 80.3a & 87.7 & 84.3 & 86.0a & 87.0a \\
\hline Sequence 3 & Cypern & thrin & & Profen & & & Cyperr & thrin & & Carbos & & & \\
\hline & 91.8 & 77.9 & 84.9a & 88.5 & 84.0 & $86.3 a$ & 78.3 & 71.1 & $74.7 b$ & 80.7 & 77.0 & $78.9 \mathrm{~b}$ & $81.2 \mathrm{a}$ \\
\hline Sequence 4 & Carbos & & & Carbo & & & Cyperr & thrin & & Cyperr & thrin & & \\
\hline & 80.1 & 78.0 & $79.1 b$ & 65.5 & 61.0 & $63.3 c$ & 78.0 & 72.0 & $75.0 \mathrm{~b}$ & 78.2 & 75.2 & $76.7 b$ & $73.5 b$ \\
\hline Sequence 5 & Acetan & rid & & Cyper & hrin & & Chlorf & apyr & & Profen & & & \\
\hline & 94.0 & 92.6 & $93.3 a$ & 85.1 & 81.0 & $82.1 \mathrm{a}$ & 91.0 & 88.3 & $89.7 a$ & 94.4 & 90.2 & $92.3 a$ & $89.4 a$ \\
\hline Sequence 6 & Profen & & & Spinos & & & Cyperr & thrin & & Carbos & & & \\
\hline & 86.9 & 82.1 & $84.5 a$ & 81.5 & 79.1 & $80.3 a$ & 75.8 & 73.9 & $74.9 \mathrm{~b}$ & 81.6 & 78.4 & 80.0a & $80.8 a$ \\
\hline Sequence 7 & Cypern & thrin & & Emma & ctin ber & & Indoxa & & & Profen & & & \\
\hline & 93.9 & 90.0 & 92.0a & 97.6 & 95.4 & $96.5 a$ & 95.9 & 93.6 & $94.8 \mathrm{a}$ & 94.2 & 92.0 & $93.1 a$ & $94.1 a$ \\
\hline
\end{tabular}

analysis indicated that the sequence 7 achieved the highest reduction percentages during the $2^{\text {nd }}, 3^{\text {rd }}$, and $4^{\text {th }}$ spray applications, which gave the following reduction percentages of 95.7 and $96.5,93.9$ and 94.8 , and 92.5 and 93.1 during the two seasons 2017 and 2018, respectively; in contrast, the sequence 4 achieved low efficacy in the $2^{\text {nd }}$ spray application. Also, the statistical analysis revealed that there were significant differences among tested sequences. Sequence 7 was the best and showed a significant reduction in boll infestation during both seasons 2017 and 2018, followed by sequence 5 . The lowest efficacy was obtained from sequence one followed by sequence four during the two seasons.

\section{Efficacy of tested sequences of insecticides against Earias insulana during two seasons}

The exhibited data in Table 3 show the variation between the weekly calculated reductions of the infestation percentages by the spiny bollworm, Earias insulana, when different sequences of compounds were used. Concerning the first spray application during both seasons 2017 and 2018, the sequence 7 (cypermethrin, 
Table $\mathbf{3}$ Infestation reduction percentage of Earias insulana during two seasons

\begin{tabular}{|c|c|c|c|c|c|c|c|c|c|c|c|c|c|}
\hline \multirow[t]{3}{*}{ Treatments } & \multicolumn{13}{|c|}{ Percentage of infestation reduction } \\
\hline & \multicolumn{3}{|c|}{ First spray } & \multicolumn{3}{|c|}{ Second spray } & \multicolumn{3}{|c|}{ Third spray } & \multicolumn{3}{|c|}{ Fourth spray } & \multirow{2}{*}{$\begin{array}{l}\text { General } \\
\text { mean }\end{array}$} \\
\hline & $\begin{array}{l}1^{\text {st }} \\
\text { week }\end{array}$ & $\begin{array}{l}2^{\text {nd }} \\
\text { week }\end{array}$ & Mean & $\begin{array}{l}1^{\text {st }} \\
\text { week }\end{array}$ & $\begin{array}{l}2^{\text {nd }} \\
\text { week }\end{array}$ & Mean & $\begin{array}{l}1^{\text {st }} \\
\text { week }\end{array}$ & $\begin{array}{l}2^{\text {nd }} \\
\text { week }\end{array}$ & Mean & $\begin{array}{l}1^{\text {st }} \\
\text { week }\end{array}$ & $\begin{array}{l}2^{\text {nd }} \\
\text { week }\end{array}$ & Mean & \\
\hline \multicolumn{14}{|l|}{ Season 2017} \\
\hline \multirow[t]{2}{*}{ Sequence 1} & \multicolumn{3}{|c|}{ Profenofos } & \multicolumn{3}{|c|}{ Profenofos } & \multicolumn{3}{|c|}{ Cypermethrin } & \multicolumn{3}{|c|}{ Cypermethrin } & \\
\hline & 70.2 & 67.2 & $68.7 \mathrm{c}$ & 84.5 & 79.2 & 81.9a & 78.1 & 74.4 & $76.3 b$ & 80.7 & 77.3 & $79.0 \mathrm{~b}$ & $76.5 d$ \\
\hline \multirow[t]{2}{*}{ Sequence 2} & \multicolumn{3}{|c|}{ Spinosad } & \multicolumn{3}{|c|}{ Profenofos } & \multicolumn{3}{|c|}{ Spinosad } & \multicolumn{3}{|c|}{ Carbosulfan } & \\
\hline & 87.4 & 85.1 & 86.3ab & 92.8 & 88.0 & $90.4 a b$ & 83.3 & 79.3 & $81.3 a$ & 85.4 & 82.5 & $84.0 \mathrm{a}$ & $85.5 b$ \\
\hline \multirow[t]{2}{*}{ Sequence 3} & \multicolumn{3}{|c|}{ Cypermethrin } & \multicolumn{3}{|c|}{ Profenofos } & \multicolumn{3}{|c|}{ Cypermethrin } & \multicolumn{3}{|c|}{ Carbosulfan } & \\
\hline & 93.4 & 88.8 & $91.1 \mathrm{ab}$ & 95.9 & 91.4 & $93.7 \mathrm{ab}$ & 86.0 & 85.0 & $85.5 a$ & 90.0 & 88.0 & 89.0a & $89.8 a$ \\
\hline \multirow[t]{2}{*}{ Sequence 4} & \multicolumn{3}{|c|}{ Carbosulfan } & \multicolumn{3}{|c|}{ Carbosulfan } & Cyper & thrin & & Cyper & thrin & & \\
\hline & 82.2 & 79.6 & $80.9 a$ & 76.9 & 74.3 & $75.6 c$ & 79.0 & 77.0 & $78.0 \mathrm{~b}$ & 77.9 & 75.8 & $76.9 b$ & $77.9 b$ \\
\hline Sequence 5 & Acetar & prid & & Cyper & hrin & & Chlorf & apyr & & Profen & & & \\
\hline & 94.6 & 92.5 & $93.6 a b$ & 95.0 & 91.4 & 93.2ab & 91.3 & 89.2 & $90.8 \mathrm{ab}$ & 89.0 & 85.0 & $87.0 a$ & $91.2 a b$ \\
\hline Sequence 6 & Profen & & & Spinos & & & Cyper & thrin & & Carbo & & & \\
\hline & 90.2 & 88.4 & $89.3 a$ & 85.2 & 83.2 & $84.2 \mathrm{a}$ & 83.7 & 82.3 & 83.0a & 81.1 & 77.8 & $79.5 b$ & $84.0 a$ \\
\hline Sequence 7 & Cyper & thrin & & Emma & ctin be & & Indoxa & & & Profen & & & \\
\hline & 96.0 & 95.9 & 96.0ab & 96.3 & 93.0 & $94.7 a b$ & 94.4 & 92.5 & $93.5 \mathrm{ab}$ & 92.8 & 90.3 & $91.6 a b$ & 94.0ab \\
\hline Season 2018 & & & & & & & & & & & & & \\
\hline Sequence 1 & Profen & & & Profen & & & Cyper & thrin & & Cyper & thrin & & \\
\hline & 73.6 & 68.1 & $71.4 d$ & 84.0 & 81.4 & $82.7 \mathrm{c}$ & 75.8 & 72.2 & $74.0 \mathrm{~d}$ & 85.9 & 82.0 & $84.0 \mathrm{c}$ & $78.0 d$ \\
\hline Sequence 2 & Spinos & & & Profen & & & Spinos & & & Carbo & fan & & \\
\hline & 94.7 & 92.8 & $93.8 a$ & 90.0 & 86.0 & $88.0 \mathrm{~b}$ & 84.3 & 80.2 & $82.3 c$ & 88.9 & 85.1 & $87.0 \mathrm{a}$ & $87.8 b$ \\
\hline Sequence 3 & Cyper & thrin & & Profen & & & Cyper & thrin & & Carbo & fan & & \\
\hline & 94.0 & 90.0 & $92.4 a$ & 91.8 & 89.3 & $90.6 a$ & 91.7 & 88.3 & $90.0 a$ & 93.0 & 90.0 & $91.5 a$ & $91.1 \mathrm{a}$ \\
\hline Sequence 4 & Carbo & Ifan & & Carbo & & & Cyper & thrin & & Cyper & thrin & & \\
\hline & 84.6 & 81.4 & 83.0a & 74.6 & 72.4 & $73.5 d$ & 86.5 & 83.4 & $85.0 \mathrm{c}$ & 79.2 & 76.1 & $77.7 d$ & $79.8 d$ \\
\hline Sequence 5 & Acetar & prid & & Cyper & hrin & & Chlorf & apyr & & Profen & & & \\
\hline & 96.9 & 95.0 & $96.0 a$ & 95.5 & 92.4 & $94.0 a$ & 93.3 & 92.0 & $92.7 a$ & 91.0 & 88.6 & 89.8ab & $93.1 a$ \\
\hline Sequence 6 & Profen & & & Spinos & & & Cyper & thrin & & Carbo & fan & & \\
\hline & 97.7 & 95.6 & $96.7 a$ & 82.7 & 80.8 & $81.8 a$ & 94.9 & 91.7 & $93.3 a$ & 83.1 & 81.0 & $82.1 \mathrm{a}$ & $88.5 b$ \\
\hline Sequence 7 & Cyper & thrin & & Emma & ctin be & & Indoxa & & & Profen & & & \\
\hline & 96.9 & 95.7 & $96.3 a$ & 96.5 & 95.2 & $95.9 a$ & 96.0 & 95.4 & $95.7 a$ & 95.0 & 93.4 & $94.2 a$ & $95.5 a$ \\
\hline
\end{tabular}

Means in the same column followed by the same letter are not significant at $P<0.05$

emmamectin benzoate, indoxacarb, and profenofos) achieved superior efficacy during the $1^{\text {st }}, 2^{\text {nd }}, 3^{\text {rd }}$, and $4^{\text {th }}$ spray applications, where it resulted in 96.0, 94.7, 93.5, and 91.6 and 96.3, 95.9, 95.7, and 94.2 throughout the seasons 2017 and 2018, respectively. The results indicated that the repetition of the same insecticide twice in a sequence did not show a clear effect could be increased or decreased, or no effect on the insecticide efficiency. Therefore, the application of profenofos in the $2^{\text {nd }}$ spray after the same treatment in the $1^{\text {st }}$ spray within sequence 1 improved the insecticide efficiency against the E. insulana, where the infestation reduction increased from 68.7 to $81.9 \%$ during the season 2017 and from 71.4 to $82.7 \%$ during the season 2018 , respectively. While the repetition of carbosulfan within sequence 4 for $1^{\text {st }}$ and $2^{\text {nd }}$ spray application depress the insecticide efficiency, the infestation reduction decreased from 80.9 to $75.6 \%$ and from 83.0 to $73.5 \%$ during the two seasons 2017 and 2018, respectively. Moreover, almost no effect of infestation reduction was recorded by cypermethrin repetition of application. According to the statistical analysis of the general mean reduction percentages, the 
(cypermethrin, emmamectin benzoate, indoxacarb, and profenofos) sequence 7 came first (94.0 and 95.5\%) during seasons 2017 and 2018 respectively. No significant difference was recorded between sequence 2 (spinosad, profenofos, spinosad, and carbosulfan) and sequence 6 (profenofos, spinosad, cypermethrin, and carbosulfan); both sequences (2 and 6) came 85.5 and $84.0 \%$ and 87.8 and $88.5 \%$ during the two seasons 2017 and 2018, respectively.

\section{Efficacy of tested sequences of insecticides against cotton bollworms}

To know the effect of different sequences on both bollworms P. gossypiella and E. insulana together, the infestation reduction of bollworms was calculated and their values are presented in Table 4. The repetition application of profenofos, in the $2^{\text {nd }}$ spray, increased the infestation reduction while the repetition of carbosulfan sequence 4 decreased the infestation reduction during the two seasons. Regarding the general mean, all tested sequences caused the infestation reduction percentages more than $75 \%$ throughout the two seasons. However, both sequences 7 and 5 were significantly higher in infestation reduction than that of other sequences. Consequently, the statistical analysis indicated that the sequence 1 had the lowest efficacy. The average of infestation reduction percentages of bollworms was also estimated (Table 5) for the two seasons. The data indicated that the sequence 7 showed a superior reduction in P. gossypiella (94.3\% infestation reduction) followed by sequence $5(90.6 \%)$. On the other hand, sequences 1 and 4 were the least effective, tested pesticide sequences in this respect. The average of infestation reduction for both $P$. gossypiella and E. insulana and the average values for the two seasons 2017 and 2018 are summarized in Table 5 as an infestation reduction of cotton bollworms. Using statistical analysis, the tested different compounds sequences could be grouped in two groups as follows: the first group, which included the sequences that gave more than $83 \%$ reduction namely sequences 7 ,

Table 4 Infestation reduction percentage of cotton bollworms during two seasons

\begin{tabular}{|c|c|c|c|c|c|}
\hline \multirow[t]{2}{*}{ Treatments } & \multicolumn{4}{|c|}{ Infestation reduction \% of four sprays } & \multirow[t]{2}{*}{ General mean } \\
\hline & 1 & 2 & 3 & 4 & \\
\hline \multicolumn{6}{|l|}{ Season 2017} \\
\hline Sequence 1 & $\begin{array}{l}\text { Profenofos } \\
72.7 b\end{array}$ & $\begin{array}{l}\text { Profenofos } \\
80.7 b\end{array}$ & $\begin{array}{l}\text { Cypermethrin } \\
74.0 \mathrm{~b}\end{array}$ & $\begin{array}{l}\text { Cypermethrin } \\
74.3 \mathrm{~b}\end{array}$ & $75.7 \mathrm{c}$ \\
\hline Sequence 2 & $\begin{array}{l}\text { Spinosad } \\
87.0 a\end{array}$ & $\begin{array}{l}\text { Profenofos } \\
90.5 a\end{array}$ & $\begin{array}{l}\text { Spinosad } \\
81.4 b\end{array}$ & $\begin{array}{l}\text { Carbosulfan } \\
84.2 a\end{array}$ & $85.8 a$ \\
\hline Sequence 3 & $\begin{array}{l}\text { Cypermethrin } \\
86.8 \mathrm{a}\end{array}$ & $\begin{array}{l}\text { Profenofos } \\
91.2 \mathrm{a}\end{array}$ & $\begin{array}{l}\text { Cypermethrin } \\
83.7 \mathrm{a}\end{array}$ & $\begin{array}{l}\text { Carbosulfan } \\
84.7 a\end{array}$ & $86.6 a$ \\
\hline Sequence 4 & $\begin{array}{l}\text { Carbosulfan } \\
80.5 a\end{array}$ & $\begin{array}{l}\text { Carbosulfan } \\
72.0 c\end{array}$ & $\begin{array}{l}\text { Cypermethrin } \\
80.0 \mathrm{a}\end{array}$ & $\begin{array}{l}\text { Cypermethrin } \\
77.2 \mathrm{C}\end{array}$ & $76.7 \mathrm{c}$ \\
\hline Sequence 5 & $\begin{array}{l}\text { Acetamiprid } \\
93.1 \mathrm{a}\end{array}$ & $\begin{array}{l}\text { Cypermethrin } \\
87.4 \mathrm{a}\end{array}$ & $\begin{array}{l}\text { Chlorfenapyr } \\
89.7 a\end{array}$ & $\begin{array}{l}\text { Profenofos } \\
89.1 a\end{array}$ & $89.8 a$ \\
\hline Sequence 6 & $\begin{array}{l}\text { Profenofos } \\
87.8 \mathrm{a}\end{array}$ & $\begin{array}{l}\text { Spinosad } \\
83.9 a\end{array}$ & $\begin{array}{l}\text { Cypermethrin } \\
81.7 \mathrm{a}\end{array}$ & $\begin{array}{l}\text { Carbosulfan } \\
77.9 \mathrm{c}\end{array}$ & $82.8 a$ \\
\hline Sequence 7 & Cypermethrin 93.7a & $\begin{array}{l}\text { Emmamectin benzoate } \\
95.2 \mathrm{a}\end{array}$ & $\begin{array}{l}\text { Indoxacarb } \\
93.7 a\end{array}$ & $\begin{array}{l}\text { Profenofos } \\
92.1 \mathrm{a}\end{array}$ & $93.7 a$ \\
\hline \multicolumn{6}{|l|}{ Season 2018} \\
\hline Sequence 1 & $\begin{array}{l}\text { Profenofos } \\
76.0 \mathrm{~b}\end{array}$ & $\begin{array}{l}\text { Profenofos } \\
77.9 \mathrm{~b}\end{array}$ & $\begin{array}{l}\text { Cypermethrin } \\
72.0 \mathrm{c}\end{array}$ & $\begin{array}{l}\text { Cypermethrin } \\
75.0 \mathrm{c}\end{array}$ & $75.2 \mathrm{C}$ \\
\hline Sequence 2 & $\begin{array}{l}\text { Spinosad } \\
92.9 a\end{array}$ & $\begin{array}{l}\text { Profenofos } \\
88.9 a\end{array}$ & $\begin{array}{l}\text { Spinosad } \\
81.3 \mathrm{ab}\end{array}$ & $\begin{array}{l}\text { Carbosulfan } \\
86.5 \mathrm{a}\end{array}$ & $87.4 a$ \\
\hline Sequence 3 & $\begin{array}{l}\text { Cypermethrin } \\
88.7 a\end{array}$ & $\begin{array}{l}\text { Profenofos } \\
88.5 a\end{array}$ & $\begin{array}{l}\text { Cypermethrin } \\
82.4 \mathrm{ab}\end{array}$ & $\begin{array}{l}\text { Carbosulfan } \\
85.2 \mathrm{a}\end{array}$ & $86.2 \mathrm{a}$ \\
\hline Sequence 4 & $\begin{array}{l}\text { Carbosulfan } \\
81.1 \mathrm{ab}\end{array}$ & $\begin{array}{l}\text { Carbosulfan } \\
68.4 c\end{array}$ & $\begin{array}{l}\text { Cypermethrin } \\
80.0 \mathrm{a}\end{array}$ & $\begin{array}{l}\text { Cypermethrin } \\
77.2 \mathrm{C}\end{array}$ & 76.7c \\
\hline Sequence 5 & $\begin{array}{l}\text { Acetamiprid } \\
94.7 a\end{array}$ & $\begin{array}{l}\text { Cypermethrin } \\
88.1 \mathrm{a}\end{array}$ & $\begin{array}{l}\text { Chlorfenapyr } \\
91.2 a\end{array}$ & $\begin{array}{l}\text { Profenofos } \\
91.1 \mathrm{a}\end{array}$ & $91.3 a$ \\
\hline Sequence 6 & $\begin{array}{l}\text { Profenofos } \\
90.6 a\end{array}$ & $\begin{array}{l}\text { Spinosad } \\
81.1 \mathrm{ab}\end{array}$ & $\begin{array}{l}\text { Cypermethrin } \\
84.1 \mathrm{a}\end{array}$ & $\begin{array}{l}\text { Carbosulfan } \\
81.1 \mathrm{ab}\end{array}$ & $84.2 \mathrm{a}$ \\
\hline Sequence 7 & $\begin{array}{l}\text { Cypermethrin } \\
94.2 \mathrm{a}\end{array}$ & $\begin{array}{l}\text { Emmamectin benzoate } \\
96.2 \mathrm{a}\end{array}$ & $\begin{array}{l}\text { Indoxacarb } \\
95.3 a\end{array}$ & $\begin{array}{l}\text { Profenofos } \\
93.7 a\end{array}$ & $94.9 a$ \\
\hline
\end{tabular}

Means in the same column followed by the same letter are not significant at $P<0.05$ 
Table 5 Average of infestation reduction percentage for bollworms in two cotton seasons

\begin{tabular}{llll}
\hline Treatments & $\begin{array}{l}\text { Pectinophora } \\
\text { gossypiella }\end{array}$ & $\begin{array}{l}\text { Earias } \\
\text { insulana }\end{array}$ & $\begin{array}{l}\text { Cotton } \\
\text { bollworms }\end{array}$ \\
\hline Sequences 1 & $73.7 \mathrm{~d}$ & $78.0 \mathrm{~b}$ & $75.9 \mathrm{~b}$ \\
Sequences 2 & $86.5 \mathrm{~b}$ & $86.7 \mathrm{ab}$ & $86.6 \mathrm{a}$ \\
Sequences 3 & $82.3 \mathrm{c}$ & $90.5 \mathrm{a}$ & $86.4 \mathrm{a}$ \\
Sequences 4 & $74.4 \mathrm{~d}$ & $79.0 \mathrm{~b}$ & $76.7 \mathrm{~b}$ \\
Sequences 5 & $89.0 \mathrm{a}$ & $92.2 \mathrm{a}$ & $90.6 \mathrm{a}$ \\
Sequences 6 & $81.2 \mathrm{c}$ & $86.3 \mathrm{ab}$ & $83.8 \mathrm{~b}$ \\
Sequences 7 & $93.8 \mathrm{a}$ & $94.8 \mathrm{a}$ & $94.3 \mathrm{a}$ \\
\hline
\end{tabular}

Means in the same column followed by the same letter are not significant at $P<0.05$

$5,2,6$, and 3, and the second group, which included sequence 1 and sequence 4 which gave 75.9 and $76.7 \%$ reduction.

\section{Efficiency of tested sequences on cotton yield loss}

The presented data in Table 6 show the percentages of protected bolls as a result of compound sequences spray. The highest percent of boll protection was 92.6 and 94.1\%; it was obtained by sequence 7 for the two seasons 2017 and 2018, respectively, and the lowest boll protection percent was 66.1 and $67.2 \%$ due to sequence 1 for the two seasons 2017 and 2018, respectively. On the other hand, all the tested sequences could be categorized into the following descending order: sequences $7,5,2,3$, 6,4 , and 1 .

The results in Tables 7 and 8 show the effect of the applied sequences on the loss of cotton yield. These data pointed to a highly significant difference between the untreated plots and the treated plots, and also, there was a significant difference between some of the applied sequences. In comparison to the untreated plots (loss percent $=44.6$ and $42.9 \%$ ), the lowest estimated percent of yield loss was found with sequence 7 (loss percent $=7.0$ and $6.4 \%$ ), whereas the highest loss percentage was

Table 6 Percentages of protected bolls in sequential treatments post-spray in two cotton seasons

\begin{tabular}{|c|c|c|c|c|}
\hline \multirow[t]{2}{*}{ Sequences } & \multicolumn{2}{|c|}{ Total infestation number } & \multicolumn{2}{|c|}{ General protected bolls \% } \\
\hline & Season 2017 & $\overline{\text { Season } 2018}$ & Season 2017 & Season 2018 \\
\hline Sequences 1 & 63 & 62 & 66.1 & 67.2 \\
\hline Sequences 2 & 31 & 27 & 83.6 & 85.5 \\
\hline Sequences 3 & 37 & 35 & 80.4 & 81.2 \\
\hline Sequences 4 & 41 & 40 & 78.0 & 78.8 \\
\hline Sequences 5 & 26 & 21 & 86.2 & 88.7 \\
\hline Sequences 6 & 35 & 32 & 81.5 & 82.1 \\
\hline Sequences 7 & 14 & 11 & 92.6 & 94.1 \\
\hline Control & 189 & 186 & - & - \\
\hline
\end{tabular}

Table 7 Assessment of loss due to bollworms in different sequence treatments during season 2017

\begin{tabular}{clllllllll}
\hline Sequences & $A$ & $B$ & $C$ & $D$ & $E$ & $F$ & $G$ & $H$ & I \% \\
\hline Sequences 1 & 60.1 & 4.7 & 1.4 & 0.24 & 2.7 & 69.1 & 61.0 & 8.1 & $11.7 \mathrm{~d}$ \\
Sequences 2 & 59.7 & 4.8 & 3.1 & 0.28 & 4.1 & 72.0 & 61.1 & 10.9 & $15.1 \mathrm{c}$ \\
Sequences 3 & 62.0 & 4.3 & 1.6 & 0.22 & 2.6 & 70.7 & 64.1 & 6.6 & $9.3 \mathrm{e}$ \\
Sequences 4 & 56.5 & 9.0 & 4.4 & 0.66 & 8.2 & 78.8 & 65.9 & 12.9 & $16.4 \mathrm{c}$ \\
Sequences 5 & 50.8 & 13.4 & 7.4 & 0.70 & 8.4 & 80.7 & 63.0 & 17.7 & $21.9 \mathrm{~b}$ \\
Sequences 6 & 59.0 & 5.7 & 3.3 & 0.44 & 6.6 & 75.0 & 63.6 & 11.4 & $15.2 \mathrm{c}$ \\
Sequences 7 & 63.2 & 4.1 & 1.7 & 0.18 & 2.2 & 71.4 & 66.4 & 5.0 & $7.0 \mathrm{e}$ \\
Control & 29.1 & 5.2 & 10.3 & 15.3 & 7.3 & 67.2 & 37.2 & 30.0 & $44.6 \mathrm{a}$ \\
\hline
\end{tabular}

detected for sequence 5 (loss percent $=21.9$ and 20.4\%). The superiority in yield loss reduction can arrange in the following order: sequences $1,2,3,4,5,6$, and 7 , respectively.

\section{Discussion}

In general, the aforementioned results, either in the pink bollworm or in the spiny bollworm, demonstrate that similar effect was obtained when such sequences were applied, according to the average of infestation reduction of both bollworms $P$. gossypiella and E. insulana for the two seasons 2017 and 2018. The tested different compounds' sequences could be grouped in two groups as follows: the first group, which included the sequences that gave more than $83 \%$ reduction namely sequences 7 , $5,2,6$, and 3, and the second group, which included sequence 1 and sequence 4 , which gave 75.9 and $76.7 \%$ reduction. According to significant differences, the data clearly show that starting and mediating the sequence with new insecticides emmamectin benzoate, indoxacarb acetamiprid, and chlorfenapyr (sequence 7 and 5) gave high infestation reduction percentages compared to other tested sequences. In similar, starting and mediating the sequence with bio-insecticide, spinosad (sequence 2), induced the efficiency of that sequence against $P$. gossypiella and E. insulana. Accordingly, this

Table 8 Assessment of loss due to bollworms in different sequence treatments during season 2018

\begin{tabular}{clllllllll}
\hline Sequences & $A$ & $B$ & $C$ & $D$ & $E$ & $F$ & $G$ & $H$ & $1 \%$ \\
\hline Sequences 1 & 60.8 & 4.8 & 2.0 & 0.20 & 3.1 & 71.0 & 63.6 & 7.4 & $10.4 \mathrm{~d}$ \\
Sequences 2 & 60.1 & 5.7 & 2.8 & 0.26 & 3.7 & 72.6 & 63.0 & 9.6 & $13.2 \mathrm{c}$ \\
Sequences 3 & 61.9 & 4.4 & 1.7 & 0.18 & 2.7 & 70.9 & 65.9 & 5.0 & $7.1 \mathrm{e}$ \\
Sequences 4 & 57.5 & 7.0 & 3.7 & 0.33 & 4.5 & 73.0 & 62.0 & 11.0 & $15.0 \mathrm{c}$ \\
Sequences 5 & 52.9 & 11.1 & 5.3 & 0.50 & 6.0 & 75.8 & 60.3 & 15.5 & $20.4 \mathrm{~b}$ \\
Sequences 6 & 59.3 & 6.2 & 3.0 & 0.29 & 4.0 & 72.8 & 62.4 & 10.4 & $14.3 \mathrm{c}$ \\
Sequences 7 & 61.6 & 4.3 & 1.9 & 0.17 & 2.6 & 70.6 & 66.1 & 4.5 & $6.4 \mathrm{e}$ \\
Control & 27.0 & 4.8 & 10.5 & 14.0 & 6.7 & 63.0 & 36.0 & 27.0 & $42.9 \mathrm{a}$ \\
\hline
\end{tabular}


result revealed to be used to control the cotton bollworms, where a new chemical group of pests has novel modes of action, which proved their efficacy against cotton bollworms with the highest seed cotton yield. Similar results were elucidated by Allen et al. (1997) who found that large- and medium-sized bollworm larvae were significantly less numerous in cyfluthrin- and spinosadtreated plots than in control plots. Tadros (2003) reported that Achook, as a botanical insecticide, resembles the efficiency of the used synthetic insecticides showing a promising way in the control program of both cotton bollworms. Shashikant (2007) noticed the efficacy of spinosad and indoxacarb against bollworm and higher seed cotton. Starting and mediating the sequence with cypermethrin (sequence 3) gave high infestation reduction percentages and seed cotton yield compared to other tested sequences with profenofos and carbosulfan (sequence 4 and 1). This finding is following that reported by El-Gogary (1987), who said that starting the program with organophosphate followed by pyrethroids gave less satisfactory results. El-Feel et al. (1991) reported that starting the controlling program with synthetic pyrethroids induced the highest reduction of the bollworms infestation. Abd El-Mageed et al. (2007) revealed that obtained data for the tested programs reduced the rate of pink bollworm and spiny bollworm larvae during the three sprays. Imran et al. (2017) reported that Bt cotton was associated with decreases in the number of insecticide sprays which caused the reduction of the risk of resistance of pink bollworm. Also, Awad et al. (2014) reported that the synthetic pyrethroids induced the most significant reduction in bollworms infestation that was associated with the highest amount of seed cotton yield more compared to the organophosphorous. In contrast, El-Feel et al. (1993) mentioned that pyrethroids could not be able to reduce the building-up of the bollworm population at the early season, while at the late season this aim is not considered.

\section{Conclusions}

As a conclusion, it can be asserted that this study points out to minimize repetition of insecticide application in the same season; furthermore, the importance of sequence insecticide programs included new groups of insecticides to directing tactics to fight against resistance development for this conventional insecticide where acetamiprid, chlorfenapyr, indoxacarb, emmamectin benzoate, and spinosad could be used in sequence programs to restore cypermethrin, profenofos, and carbosulfan susceptibility.

These findings may have considerable practical implications for Pectinophora gossypiella and Earias insulana resistance management, and this leads to obtaining the optimum regimen of insecticides, which gives the reduction of cotton bollworms infestation and high cotton yield.
Author's contributions

The author contributed to the production and writing of the manuscript. The author read and approved the final manuscript.

Funding

There was no funding for this work.

Availability of data and materials

All data generated during this study are included in this published article.

Ethics approval and consent to participate

The manuscript does not contain any studies involving human participants, human data, or human tissue.

Consent for publication

Not applicable.

\section{Competing interests}

The author declares that he/she has no competing interests.

Received: 13 July 2019 Accepted: 10 September 2019

Published online: 12 October 2019

References

Abd El-Mageed AEM, Anwar EM, Elgohary LRA, Dahi HF (2007) Evaluation of several programs of sequences pesticides application on cotton bollworms and some other sucking pests in cotton field. J Entomol. 4:93-103

Allen CT, Frizzell S, Earnest L, Tugwell P, Haynes J, Riddle S, Scott K (1997) Bollworm control test. In: Special Report Agriculture Experiment Station, Division of Agriculture, Univ. Arkansas, vol 183, pp 184-187

Amin AA, Gergis MO (2006) Integrated management strategies for control of cotton key pests in Middle Egypt. Agron Res 4(Special issue):121-128

Awad HA, El-Naggar AZ, El-Bassouiny HM, Haity M, Tadros (2014) Evaluation of certain insect growth regulators and some insecticides against the cotton leafworm and bollworms in field cotton and their effect on yield. Alex Sci Exch J 35:80-86

El-Feel EA, El-Sorady AEM, Awad HA, Omar ME (1993) Influence of certain insecticide against bollworms and other cotton pests and their predators. Alex Sci Exch. 14:145-165

El-Feel EA, Khidr AA, Abou-Kahla MM, Abbass MG (1991) Effect of some insecticidal sequence, time intervals between sprays and early spray on the pink bollworm, Pectinophora gossypiella (Saund.) and cotton yield. Egypt J Agric Res. 69:73-82

El-Gogary OA (1987) Effect of phermones and chemical pesticides on population density of cotton bollworms. In: Ph.D. Thesis, Fac. Agric., Helwan Univ.

Gunning RV, Devonshire AL (2002) Negative cross resistance indoxacarb and pyrethroids in Australian Helicoverpa armigera: a tool for resistance management. Resist Pest Manag. 11:52

Hassan EMA (2007) Assessment of losses in cotton yield due to the infestation of bollworms with reference to determination of the economic injury levels, M. Sc. In: Thesis Faculty of Agric. Alex. Univ

Henderson CF, Tilton WE (1955) Tests with acaricides against the brown wheat mite. J Econ Entomol. 48:157-161

Imran AR, Tajwer SS, Arfan AG, Aaha MA, Fahad NK, Ghulam HA, Maqsood AR (2017) Effect of different synthetic pesticides against pink bollworm Pectinophora gossypiella (Saund.) on Bt. and non-Bt. cotton crop. J Basic Applied Sci. 13:454-458

Ramsubramanian T (2004) Magnitude, mechanism and management of pyrethroids resistance in Helicoverpa armigera (Hubner) in India. J Entomol. 1:6-11

Shashikant SU (2007) Bio-efficacy of newer insecticides against cotton bollworm. Karnataka J Agric Sci. 20:648-650

Tadros HM (2003) Effect of some insecticides on cotton bollworms and on quantity and quality of cotton yield. In: M.Sc. Fac. of Agric. (Saba-Bacha), Alex. Univ., Alexandria. Egypt

\section{Publisher's Note}

Springer Nature remains neutral with regard to jurisdictional claims in published maps and institutional affiliations. 